This document is the Accepted Manuscript version of a Published Work that appeared in final form in Journa of the American Chemical Society, copyright (C) American Chemical Society after peer review and technical editing by the publisher. To access the final edited and published work see http://pubs.acs.org/doi/abs/10.1021/ja509077a

\title{
Ligand-controlled Regiodivergent Ni-Catalyzed Reductive Carboxylation of Allyl Esters with $\mathrm{CO}_{2}$ Toni Moragas ${ }^{\dagger \ddagger}$, Josep Cornella ${ }^{\dagger \ddagger}$ and Ruben Martin ${ }^{* \dagger \$}$
}

\author{
${ }^{\dagger}$ Institute of Chemical Research of Catalonia (ICIQ), Av. Països Catalans 16, 43007, Tarragona, Spain \\ ${ }^{\S}$ Catalan Institution for Research and Advanced Studies (ICREA), Passeig Lluïs Companys, 23, 08010, Barcelona, Spain
}

\section{Supporting Information Placeholder}

ABSTRACT: A novel Ni-catalyzed regiodivergent reductive carboxylation of allyl esters with $\mathrm{CO}_{2}$ has been developed. This mild, user-friendly and operationallysimple method is characterized by an exquisite selectivity profile that is dictated by the ligand backbone.

The ability to control the outcome of catalytic reactions by the fine-tuning of the catalyst structure is central in the cross-coupling arena. ${ }^{1}$ Despite the advances realized, the development of catalytic regiodivergent protocols from a common precursor in a rational and predictable manner remains a formidable challenge, ${ }^{2}$ thus offering a unique opportunity to improve our ever-growing chemical portfolio. Intriguingly, while allyl electrophiles have been successfully employed as coupling partners with nucleophilic counterparts, ${ }^{3}$ the utilization of these motifs in catalytic reductive protocols is not as commonly practiced as one might anticipate. ${ }^{4,5}$ This is probably due to the difficulty for discriminating at will both ends of the initially generated $\pi$-allyl metal complex, ${ }^{3}$ resulting in regioselectivity issues (Scheme 1, II vs III). Indeed, a catalyst-controlled regiodivergent reductive event for selectively obtaining II and III from a common allyl electrophile (I) remains an unexplored area of research.

Scheme 1. Regiodivergency in Allyl Electrophiles

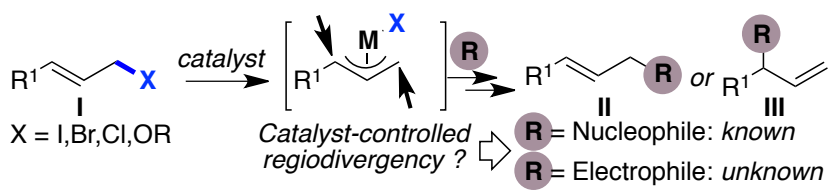

Carbon dioxide $\left(\mathrm{CO}_{2}\right)$ has emerged as a powerful synthon and renewable chemical feedstock for organic synthesis. ${ }^{6}$ The interest for designing new catalytic reactions using $\mathrm{CO}_{2}$ arises from its low cost, high abundance and lack of toxicity and flammability. Nonetheless, the design of catalytic processes based on carbon dioxide is particularly challenging since $\mathrm{CO}_{2}$ is kinetically inert and not particularly soluble in commonly employed organic solvents at atmospheric pressure, thus resulting in competitive side-reactions. In recent years, $\mathrm{we}^{7}$ and oth$\mathrm{ers}^{8}$ launched a program to unravel the potential of cata- lytic reductive carboxylation events using aryl or alkyl electrophiles en route to carboxylic acids, privileged motifs in a wide variety of pharmaceuticals and agrochemicals. ${ }^{9}$ Although these reactions have reached remarkable levels of sophistication, ${ }^{7,8}$ a ligand-controlled selectivity in carboxylation events is unknown, leaving ample opportunities to improve upon existing carboxylation techniques. Herein, we summarize our investigations aiming at the development of an unprecedented regiodivergent catalytic reductive carboxylation strategy (Scheme 2). ${ }^{10}$ The protocol is inherently modular, allowing for the introduction of the carboxylic motif at any site of the allyl terminus depending on the ligand employed (paths a \& b). To the best of our knowledge, this constitutes the first time that the nature of the ligand dictates the outcome of carboxylation events. ${ }^{11}$ The transformation is mild and user-friendly, constituting an added value when compared with classical techniques based on well-defined allyl organometallic species, ${ }^{12,13}$ halide counterparts and/or high $\mathrm{CO}_{2}$ pressures.

Scheme 2. Regiodivergent Catalytic Carboxylation

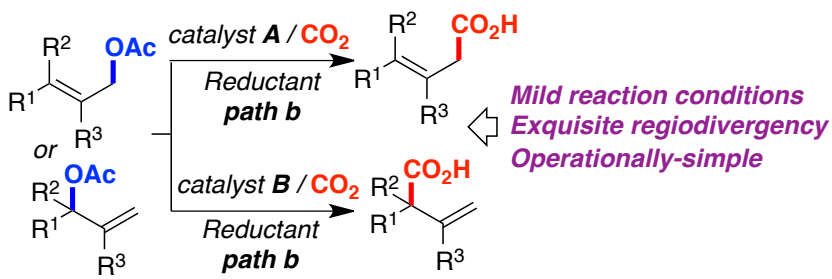

We started our investigations using 1a as the model substrate and the influence of all reaction components was systematically examined. As for other carboxylation reactions, ${ }^{7,8}$ we anticipated that the efficiency of the reaction would be strongly ligand dependent. As shown in Table 1, this was indeed the case. After some experimentation, ${ }^{14,15}$ we found that $\mathrm{C} 2$-substituted bipyridine $\mathbf{L 2}$ in DMF and $\mathrm{Mn}$ as reductant at atmospheric $\mathrm{CO}_{2}$ pressure was particularly suited for our purposes (entry 2). More importantly, such seemingly trivial modification at $\mathrm{C} 2$ was critical for improving the reactivity and selectivity pattern (entry 1 vs 2). Although L3 and L4 resulted in a decrease of selectivity (entry 3 ), a survey of additives revealed that both reactivity and $\mathbf{2 a : 3 a}$ ratio could be accentuated by adding $\mathrm{MgCl}_{2}$ with $\mathbf{L 3},{ }^{16}$ afford- 
ing exclusively 2a in $77 \%$ isolated yield at $5 \mathrm{~mol} \%$ catalyst loading (entry 4). ${ }^{17,18}$ Intriguingly, the use of $\mathrm{MgCl}_{2}$ did not have any influence for $\mathbf{L} \mathbf{2}$, thus showing the subtleties of our system. Strikingly, the use of commercially available quaterpyridine $\mathbf{L 5}$ resulted in a selectivity switch under identical reaction conditions, favouring the formation of 3a, albeit in lower yields (entry 6). These results tacitly suggest that the ligand backbone exclusively dictates the selectivity pattern. The fine-tuning of the Ni:L5 ratio, reductant, solvent and the inclusion of $\mathrm{Na}_{2} \mathrm{CO}_{3}$ as additive allowed for obtaining 3a in $72 \%$ isolated yield with an excellent 3a:2a ratio (entry 9). ${ }^{17,18}$ While similar selectivity was observed for L6 and L7 (entries 10 and 11), the best results were found with $\mathbf{L 5}$. As anticipated, control experiments revealed that all reaction components were crucial for success. ${ }^{14}$ Taking into consideration the lack of precedents when using $\mathbf{L 5}$ in the cross-coupling arena, we anticipate that $\mathbf{L 5}$ might open up perspectives in ligand design for effecting otherwise inaccessible coupling processes.

Table 1. Optimization of the Reaction Conditions ${ }^{a}$

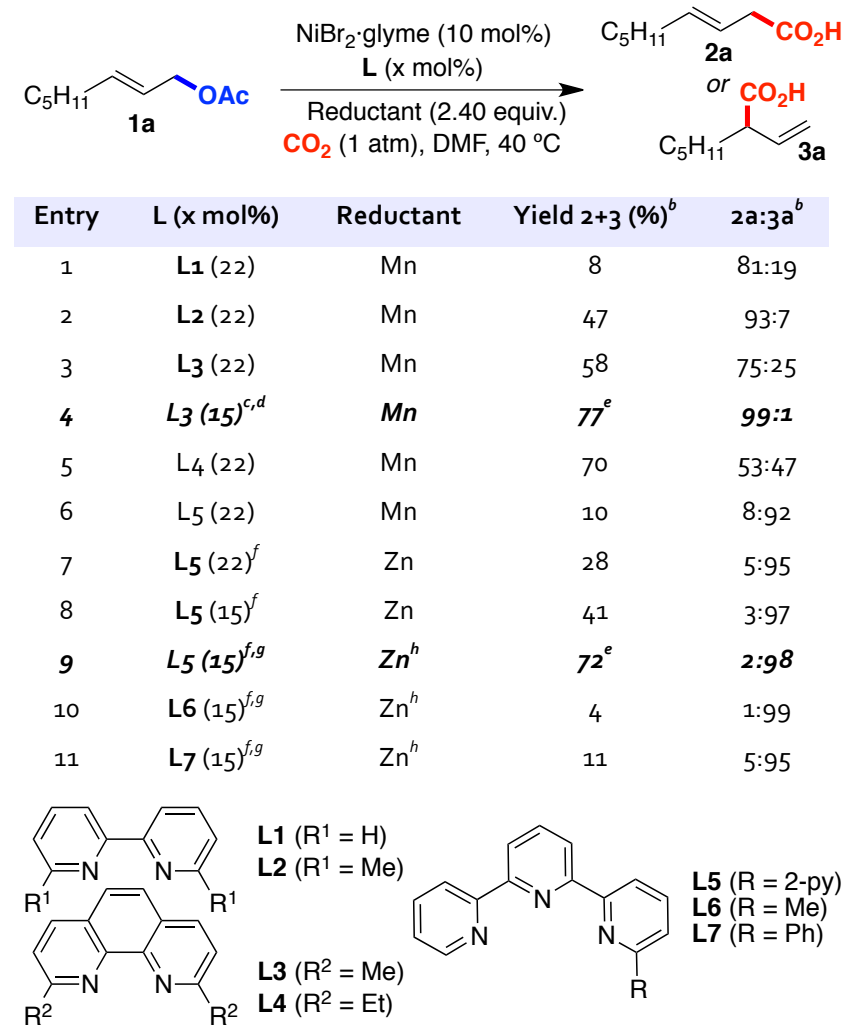

${ }^{a}$ 1a $(0.25 \mathrm{mmol}), \mathrm{NiBr}_{2} \cdot$ glyme $(10 \mathrm{~mol} \%), \mathbf{L}(\mathrm{x} \mathrm{mol} \%)$, reductant (2.40 equiv.), DMF $(0.17 \mathrm{M}), \mathrm{CO}_{2}(1 \mathrm{~atm})$ at 40 ${ }^{\circ} \mathrm{C}$ for $16 \mathrm{~h} .{ }^{b}$ Determined by GC using anisole as internal standard. ${ }^{c} \mathrm{MgCl}_{2}$ (2 equiv.) was added. ${ }^{d} \mathrm{NiBr}_{2} \cdot$ glyme (5 mol\%). ${ }^{e}$ Isolated yield. ${ }^{f}$ DMA (0.17 M). ${ }^{g} \mathrm{Na}_{2} \mathrm{CO}_{3}(20$ mol\%) was added. ${ }^{h} \mathrm{Zn}$ (1.75 equiv.).

Table 2. Ligand-controlled Regiodivergent Carboxylation

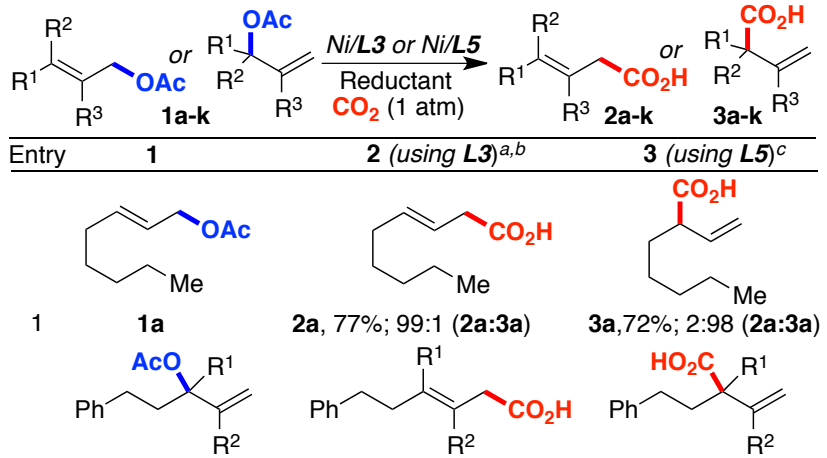

2 1b, $\mathrm{R}^{1}=\mathrm{R}^{2}=\mathrm{H} \quad$ 2b, $84 \% ; 97: 3(\mathbf{2 b}: \mathbf{3 b})^{d} \quad$ 3b, $71 \% ; 6: 94$ (2b:3b)

3 1c, $R^{1}=H ; R^{2}=M e \quad 2 c, 59 \% ; 97: 3(2 c: 3 c)^{e} \quad 3 c, 60 \% ; 1: 99(2 c: 3 c)$

4 1d, $R^{1}=\mathrm{Me} ; \mathrm{R}^{2}=\mathrm{H} \quad$ 2d, 57\%; $97: 3$ (2d:3d) $)^{e, f} \quad$ 3d, 55\%; 3:97 (2d:3d)

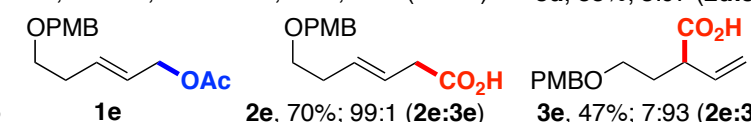

$5 \quad 1$ e 2e, $70 \% ; 99: 1(2 e: 3 e) \quad 3 e, 47 \% ; 7: 93(2 e: 3 e)$

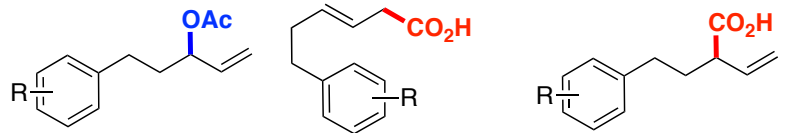

6 1f, $\mathrm{R}=3-\mathrm{CO}_{2} \mathrm{Et} \quad$ 2f, $52 \% ; 99: 1(\mathbf{2 f : 3 f})^{g} \quad$ 3f, $57 \% ; 7: 93$ (2f:3f)

$7 \mathbf{1 g}, \mathrm{R}=3-\mathrm{Cl} \quad \mathbf{2 g}, 63 \% ; 90: 10(\mathbf{2 g}: \mathbf{3 g}) \quad \mathbf{3 g}, 64 \% ; 7: 93(\mathbf{2 g}: \mathbf{3 g})$

$8 \quad \mathbf{1 h}, \mathrm{R}=2-\mathrm{SMe} \quad 2 \mathrm{~h}, 58 \% ; 82: 18(\mathbf{2 h}: 3 \mathrm{~h})^{g} \quad$ 3h, 52\%; 5:95 (2h:3h)

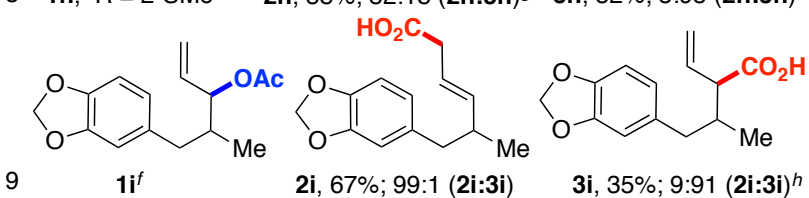

$9 \quad \mathbf{1 i}^{f} \quad \mathbf{2 i}, 67 \% ; 99: 1(\mathbf{2} \mathbf{i}: 3 \mathbf{i}) \quad 3 \mathbf{i}, 35 \% ; 9: 91(\mathbf{2 i}: 3 \mathbf{i})^{h}$
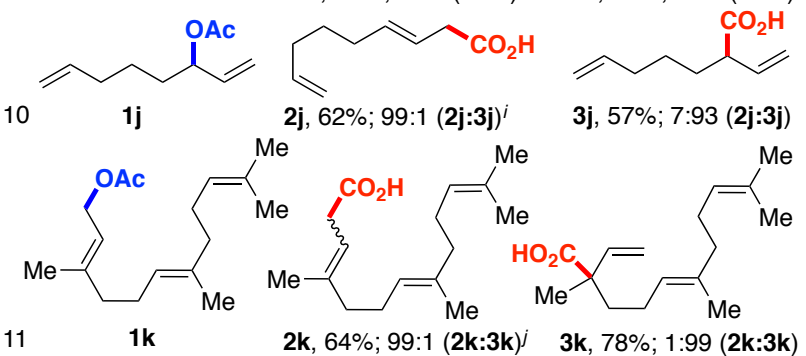

${ }^{a}$ Using L3: 1 (0.25 mmol), $\mathrm{NiBr}_{2} \cdot$ glyme (5 mol\%), L3 (15 $\mathrm{mol} \%), \mathrm{Mn}(0.60 \mathrm{mmol}), \mathrm{MgCl}_{2}(0.50 \mathrm{mmol})$ in DMF at 40 ${ }^{\circ} \mathrm{C} .{ }^{b} \mathbf{2 a}-\mathbf{2 j}$ were obtained in $\geq 9: 1 \mathrm{E}: Z$ ratio. ${ }^{c}$ Using $\mathbf{L 5}: \mathbf{1}$ (0.25 mmol), $\mathrm{NiBr}_{2} \cdot$ glyme (10 mol\%), L5 (15 mol\%), Zn (0.44 mmol), $\mathrm{Na}_{2} \mathrm{CO}_{3}(20 \mathrm{~mol} \%)$ in DMA at $40{ }^{\circ} \mathrm{C} .{ }^{d}$ At 50 ${ }^{\circ} \mathrm{C}$. ${ }^{e} \mathrm{NiBr}_{2} \cdot$ glyme $(10 \mathrm{~mol} \%)$ at $60{ }^{\circ} \mathrm{C} .{ }^{f} 1.5: 1$ (E:Z). ${ }^{g}$ $\mathrm{NiBr}_{2} \cdot \operatorname{glyme}(10 \mathrm{~mol} \%)$ and $\mathbf{L} \mathbf{4}(30 \mathrm{~mol} \%) .{ }^{h} 1: 1$ syn:anti. $\mathrm{NiBr}_{2} \cdot$ glyme (3 mol\%). ${ }^{j}$ 2.3:1 (E:Z).

Encouraged by these precedents, we turned our attention to the preparative scope of our $\mathrm{Ni}$-catalyzed regiodivergent carboxylation protocol (Table 1). As shown, a variety of allyl acetates were all carboxylated in good yields and excellent regioselectivities depending on the ligand utilized. As expected, the carboxylation strategy based on $\mathbf{L 3}$ resulted in the predominant formation of $E$ configured isomers (2a-k). ${ }^{19}$ Remarkably, a high selectivity profile was obtained regardless of whether linear or $\alpha$-branched allyl acetates were utilized. These results reinforce the notion that our regiodivergent protocol does not operate under substrate-control and that the ligand exclusively dictates the selectivity pattern. As 
shown for 1c-1d, the inclusion of substituents on the allyl motif did not have a deleterious effect on selectivity. The preparation of carboxylic acids bearing a quaternary center (3d and $\mathbf{3 k}$ ) is particularly noteworthy since Ni-catalyzed reductive coupling reactions of tertiary alkyl electrophiles are virtually inexistent. ${ }^{20}$ The chemoselectivity profile of our method is further illustrated by the presence of ethers (1e), acetals (1i), esters (1f), thioethers $(\mathbf{1 h})$ or alkenes $(\mathbf{1 j}$ and $\mathbf{1 k})$. Strikingly, while the inclusion of thioether motifs in the side chain had a negative impact for $\mathbf{2 h}$, no erosion in selectivity was found when operating under a $\mathbf{L 5}$ regime, hence suggesting that thioethers compete with substrate binding with $\mathbf{L 3}$. Interestingly, the selectivity towards $\mathbf{3 i}$ was not affected by substituents in the $\alpha$ position of the allyl acetate fragment (1i), albeit $\mathbf{3 i}$ was obtained in lower yield. $^{21}$ The successful preparation of $\mathbf{2 k}$ and $\mathbf{3 k}$ from naturally ocurring farnesyl acetate $1 \mathbf{k}$ highlights the robustness of our protocol in the presence of multiple double bonds. Moreover, the carboxylation could be conducted without affecting the aryl chloride entity, providing an additional functional handle via cross-coupling techniques (2g and $\mathbf{3 g}$ ). Importantly, we found that the carboxylation of $\mathbf{1} \mathbf{j}$ could be conducted without noticeable 5-exo-trig cyclization $(\mathbf{2} \mathbf{j}$ and $\mathbf{3} \mathbf{j}){ }^{22}$ Overall, the data in Table 2 demonstrates the robustness and prospective impact of our regiodivergent carboxylation protocol.

\section{Scheme 3. Convergent Synthesis of 21 and 31 from 1l-1n}

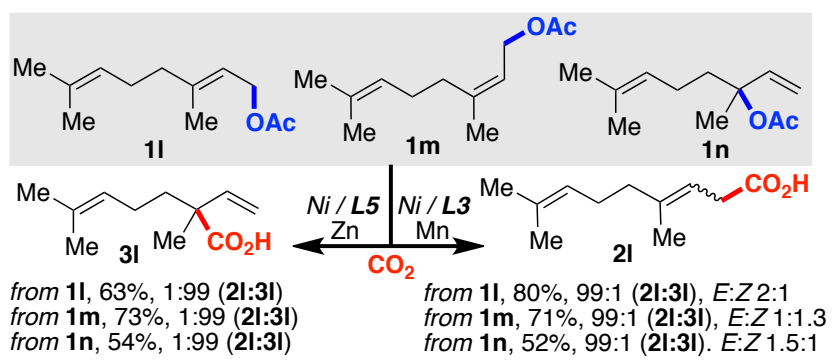

Guided by the assumption that the reaction might not be substrate-controlled, we speculated that a different set of constitutional and configurational isomers could converge to a single carboxylic acid with a protocol based on $\mathbf{L 3}$ and L5. In line with our expectations, 11-1n were exclusively converted into either $\mathbf{2 l}$ or $\mathbf{3 l}$ in good yields with variable $E / Z$ ratios (Scheme 3). ${ }^{19}$ We believe these results suggest common reaction intermediates ${ }^{23}$ and increase the flexibility in synthetic design for preparing carboxylic acids from different precursors. Although a mechanistic study should await further investigations, we set out to explore the intermediacy of $\mathbf{L 3}$ - and $\mathbf{L 5}-\mathrm{Ni}$ complexes. Following a procedure described by Nocera, ${ }^{24}$ we prepared air-sensitive $\mathbf{4}$ and 5 by reacting $\mathbf{L} 3$ or $\mathbf{L 5}$ with $\mathrm{Ni}(\mathrm{COD})_{2}$ in THF and their structures were univocally characterized by X-ray crystallography (Scheme 4). ${ }^{14,25}$ Intriguingly, while 2 a could only be obtained in the presence of a reducing agent by using 4 , 3a was cleanly produced with $\mathbf{5}$, even in the absence of reductant. ${ }^{26}$ These experiments confirm that the ligand backbone dictates the selectivity pattern and strongly suggest a different mechanistic pathway for $\mathbf{L 5}$ that differs from other reductive coupling events. At present, we believe that $\mathbf{L 5}$ might behave similarly to pincer-type ligands in related carboxylation events via $\eta^{1}$-allyl intermediates $^{27}$ and that the additional pyridine motif might be acting as a hemilabile ligand, thus tempering the catalytic activity on the $\mathrm{Ni}$ center and preventing decomposition pathways.

\section{Scheme 4. Stoichiometric Experiments}
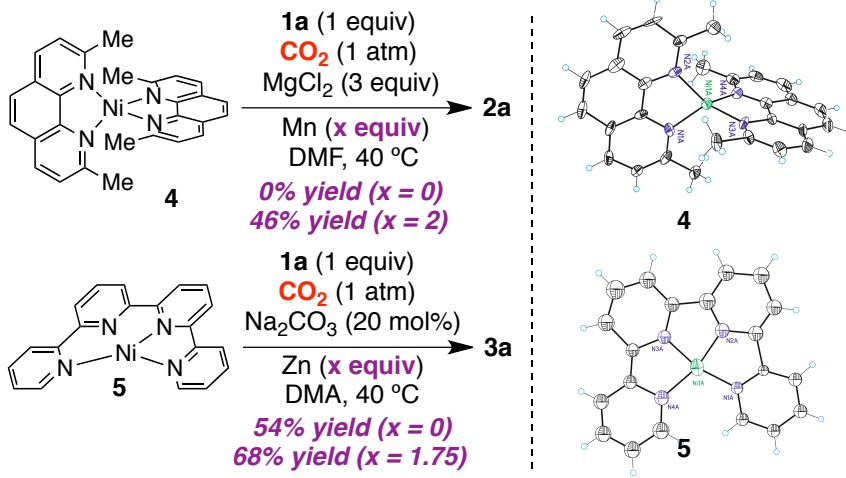

In summary, we have described a novel, mild and userfriendly Ni-catalyzed regiodivergent carboxylation of allyl acetates with $\mathrm{CO}_{2}$. This protocol constitutes the first regiodivergent catalytic reductive coupling of allyl electrophiles and provides consistent evidence that the ligand dictates the selectivity pattern. We anticipate that this study will lead to new knowledge in catalyst design by using unconventional ligand backbones. Further investigations into the mechanism and the development of an asymmetric version are currently underway.

\section{ASSOCIATED CONTENT}

Supporting Information. Experimental procedures and spectral data. This material is available free of charge via the Internet at http://pubs.acs.org.

\section{AUTHOR INFORMATION}

Corresponding Author

* rmartinromo@iciq.es

Author contributions

$\$$ These authors contributed equally to this work

Funding Sources

No competing financial interests have been declared.

\section{ACKNOWLEDGMENT}

We thank ICIQ, the European Research Council (ERC277883) and MINECO (CTQ2012-34054 \& Severo Ochoa Excellence Accreditation 2014-2018; SEV-2013-0319) for support. Johnson Matthey, Umicore and Nippon Chemical Industrial are acknowledged for a gift of metal \& ligand sources. J.C thanks European Union (FP7-PEOPLE-2012- 
IEF-328381) for a fellowship. We thank Prof. V. Grushin \& Prof. S. Ogoshi for useful discussions and Eduardo Escudero for all X-Ray crystallographic data. This paper is dedicated to the memory of Prof. Gregory L. Hillhouse.

\section{REFERENCES}

(1) Diederich, F.; Meijere, A., Eds. Metal-Catalyzed CrossCoupling Reactions; Wiley-VCH: Weinheim, 2004.

(2) For selected reviews: (a) Mahatthananchai, J.; Dumas, A. M.; Bode, J. W. Angew. Chem., Int. Ed. 2012, 51, 10954. (b) Afagh, N. A.; Yudin, A. K. Angew. Chem., Int. Ed. 2010, 49, 262. (c) Trost, B. M. Science 1983, 219, 245.

(3) For selected reviews on metal-catalyzed allylic substitution: (a) Arnold, J. S.; Zhang, Q.; Nguyen, H. M. Eur. J. Org. Chem. 2014, 23, 4925. (b) Sundararaju, B.; Achard, M.; Bruneau, C. Chem. Soc. Rev. 2012, 41, 4467. (c) Helmchen, G.; Dahnz, A.; Dübon, P.; Schelwies, M.; Weihofen, R. Chem. Commun. 2007, 675. (d) Trost, B. M.; Crawley, M. L. Chem. Rev. 2003, 103, 2921.

(4) For reviews on metal-catalyzed reductive couplings: (a) Moragas, T.; Correa, A.; Martin, R. Chem. Eur. -J. 2014, 20, 8242. (b) Tasker, S. Z.; Standley, E. A.; Jamison, T. F. Nature 2014, 509, 299. (c) Everson, D. A.; Weix, D. J. J. Org. Chem. 2014, 79, 4793. (d) Knappe, C. E. I.; Grupe, S.; Gärtner, D.; Corpet, M.; Gosmini, C.; Jacobi von Wangelin, A. Chem. Eur. -J. 2014, 20, 6828.

(5) For selected regioselective (not regiodivergent) reductive couplings of allyl electrophiles: (a) Tan, Z.; Wan, X.; Zang, Z.; Qian, Q.; Deng, W.; Gong, H. Chem. Commun. 2014, 50, 3827. (b) Anka-Lufford, L. L.; Prinsel, M. R.; Weix, D. J. J. Org. Chem. 2012, 77, 9989. (c) Wang, S.; Qian, Q.; Gong, H. Org. Lett. 2012, 14, 3352. (d) Durandetti, M.; Nedelec, J. -Y.; Perichon, J. J. Org. Chem. 1996, 61, 1748.

(6) For selected reviews: (a) Zhang, L.; Hou, Z. Chem. Sci. 2013, 4, 3395. (b) Tsuji, Y.; Fujihara, T. Chem. Commun. 2012, 48, 9956. (c) Cokoja, M.; Bruckmeier, C.; Rieger, B.; Herrmann, W. A.; Kuhn, F. E. Angew. Chem., Int. Ed. 2011, 50, 8510. (d) Huang, K.; Sun, C. -L.; Shi, Z. -J. Chem. Soc. Rev. 2011, 40, 2435. (e) Martin, R.; Kleij, A. W. ChemSusChem. 2011, 4, 1259.

(7) (a) Liu, Y.; Cornella, J.; Martin, R. J. Am. Chem. Soc. 2014, 136, 11212. (b) Correa, A.; Leon, T.; Martin, R. J. Am. Chem. Soc. 2014, 136, 1062. (c) Leon, T.; Correa, A.; Martin, R. J. Am. Chem. Soc. 2013, 135, 1221. (d) Correa, A.; Martin, R. J. Am. Chem. Soc. 2009, 131, 15974.

(8) (a) Nogi, K.; Fujihara, T.; Terao, J.; Tsuji, Y. Chem. Commun. 2014, 50, 13052. (b) Tran-Vu, H.; Daugulis, O. ACS Catal. 2013, 3, 2417. (c) Fujihara, T.; Nogi, K.; Xu, T.; Terao, J.; Tsuji, Y. J. Am. Chem. Soc. 2012, 134, 9106.

(9) Maag, H. Prodrugs of Carboxylic Acids; Springer: New York, 2007.

(10) For selected metal-catalyzed regiodivergent reductive coupling processes not involving $\mathrm{CO}_{2}$ or allyl electrophiles: (a) Zhao, Y.; Weix, D. J. J. Am. Chem. Soc. 2014, 136, 48. (b) Köpfer, A.; Sam, B.; Breit, B.; Krische, M. J. Chem. Sci., 2013, 4, 1876. (c) Shareef, A. -R.; Sherman, D. H.; Montgomery, J. Chem. Sci. 2012, 3, 892. (d) Miller, K. M.; Jamison, T. F. J. Am. Chem. Soc. 2004, 126, 15342.

(11) This work was presented at the 19th International Symposium on Homogeneous Catalysis, Ottawa (July 6-11, 2014). At the conference, the Tsuji group described a nonreductive $\mathrm{Cu}$-catalyzed regiodivergent carboxylation of allenes using silylboranes as coupling partners.

(12) For the carboxylation of allyl boronates, see: (a) Duong, H. A.; Huleatt, P. B.; Tan, Q. -W.; Shuying, E. L. Org. Lett.
2013, 15, 4034. For the carboxylation of allyl stannanes: (b) Hruszkewycz, D. P.; Wu, J.; Hazari, N.; Incarvito, C. D. J. Am. Chem. Soc. 2011, 133, 3280. (c) Shi, M.; Nicholas, K. M. J. Am. Chem. Soc. 1997, 119, 5057.

(13) For selected carboxylation of well-defined organometallics derived from allyl halides: (a) Wu, J.; Green, J. C.; Hazari, N.; Hruszkewycz, D. P. Incarvito, C. D.; Schmeier, T. J. Organometallics 2010, 29, 6369. (b) Miao, B.; Ma, S. Chem. Commun. 2014, 50, 3285. (c) Hung, T.; Jolly, P. W.; Wilke, G. J. Organomet. Chem. 1980, 190, C5. (d) Courtois, G.; Migniac, L. J. Organomet. Chem. 1974, 69, 1. (e) Friederich, L. E.; Cormier, R. A. J. Org. Chem. 1971, 36, 3011.

(14) See Supporting information for details.

(15) No products were detected under previously reported $\mathrm{Ni}$ catalyzed reductive carboxylation reactions (ref. 7b, 7c, 8c).

(16) For the beneficial role of $\mathrm{MgCl}_{2}$ in carboxylation reactions or reductive events, see: (a) Wu, F.; Lu, W.; Qian, Q.; Ren, Q.; Gong, H. Org. Lett. 2012, 14, 3044. (b) Metzger, A.; Bernhardt, S.; Manolikakes, G.; Knochel, P. Angew. Chem., Int. Ed. 2010, 49, 4665. (c) Ref. 7c.

(17) Dimerization and reduction account for the mass balance. The inclusion of $\mathrm{H}_{2} \mathrm{O}(10-100 \mathrm{~mol} \%)$ shut down the reactivity.

(18) We found identical results when scaling up the reaction of 1 a (1 mmol) under a $\mathbf{L 3}$ or $\mathbf{L} 5$ regime.

(19) Lower $E / Z$ ratios were found for $\mathbf{2 k - n}$, an observation that is in line with the directing effect of tethered alkenes in $\mathrm{Ni}$ catalyzed coupling reactions. See for example: ref. 10d.

(20) For remarkable exceptions: (a) Luo, L.; Zhang, J. -J.; Ling, W. -J.; Shao, Y. -L.; Wang, Y. -W.; Peng, Y. Synthesis 2014, 46, 1908. (b) Yang, D.; Belardi, J. K.; Micalizio, G. C. Tetrahedron Lett. 2011, 52, 2144. (c) Refs. 7c and 8a

(21) No reaction took place when exposing 4,4-dimethylpent-1en-3-yl acetate possessing a quaternary carbon in $\alpha$-position under the conditions based upon L5. Likewise, no reaction was observed with cyclohex-2-en-1-yl acetate.

(22) At higher $\mathrm{Ni} / \mathbf{L} 3$ loadings we observed $\mathbf{2 j}$ and 5-exo-trig cyclization in a linear relationship, suggesting that a radicalescape-rebound mechanism could be operating. Indeed, the reaction of $\mathbf{1 j}$ with $\mathrm{Ni} / \mathbf{L} \mathbf{3}$ was inhibited by addition of radical scavengers such as TEMPO or galvinoxyl. Intriguingly, $\mathbf{3 j}$ was the only observable product with $\mathrm{Ni} / \mathbf{L 5}$, reinforcing the notion that a different interplay operates for $\mathbf{L 5}$.

(23) In line with this notion, we found that racemic $\mathbf{3 b}$ was obtained from $(\mathrm{R})-\mathbf{1 b}(65 \%$ ee $)$ under a $\mathrm{Ni} / \mathbf{L} 5$ regime.

(24) Powers, D. C.; Anderson, B. L.; Nocera, D. G. J. Am. Chem. Soc. 2013, 135, 18876.

(25) Taking into consideration the tetrahedral geometry for $\mathrm{Ni}(0)$ complexes, the square-planar environment found for $\mathbf{5}$ might suggest that this complex would be best described as a $\mathrm{Ni}(\mathrm{II})$ complex of a reduced quaterpyridine ligand dianion rather than a $\mathrm{Ni}(0)$ complex. For an excellent review dealing with redox-active ligands: Hu, X. Chem. Sci. 2011, 2, 1867.

(26) The use of stoichiometric $\mathrm{Ni}(\mathrm{COD})_{2} / \mathbf{L 3}$ or $\mathrm{Ni}(\mathrm{COD})_{2} / \mathbf{L 5}$ in provided otherwise identical reactivity to 4 and 5 .

(27) Tridentate, pincer-type ligands have shown to promote a related $\alpha$-branched carboxylation of allenes. The mechanism is believed to proceed via the formation of a new C-C bond between $\mathrm{CO}_{2}$ and the $\gamma$-carbon of an in situ generated $\eta^{1}$-allyl metal complexes, see: (a) Suh, H.-W.; Guard, L. M.; Hazari, N. Chem. Sci. 2014, 5, 3859. (b) Takaya, J.; Iwasawa, N. J. Am. Chem. Soc. 2008, 130, 15254. 
Ligand-controlled catalytic regiodiodivergent reductive carboxylation

$\begin{gathered}14 \text { examples } \\ \text { up to } 78 \% \text { yield } \\ \text { up to } 99: 1 \text { selectivity }\end{gathered}$
$\begin{gathered}\text { Useductant } \\ \mathrm{CO}_{2}(1 \mathrm{~atm})\end{gathered}$

